\title{
Was ist Natur?
}

Johannes Corrodi Katzenstein

Wenn wir uns der Frage "Was ist Natur?« annähern, scheint sich die Antwort vielen aufzudrängen. ${ }^{\star}$ Natur ist all das und nur das, was von den modernen Naturwissenschaften erforscht wird. Natur ist der exklusive Gegenstand der Naturwissenschaften. Ein Blick in die heutige philosophische Landschaft zeigt zudem, dass mit dieser methodologischen Überzeugung oft eine ontologische Position des Naturalismus einhergeht. Diese besagt, dass allein Natur "real « ist. ${ }^{1}$ Welche philosophischen und theologischen Perspektiven, welche Gedanken, Bilder, Assoziationen und Gefühle das Thema Natur sonst noch hervorrufen mag - etwa die Idee einer göttlichen Präsenz in der Natur oder literarische Sehnsuchtsprojektionen einer unberührten Natur -, der Sache nach scheint die Frage »Was ist Natur? " oft entschieden zu sein, sobald sie gestellt wurde. Dieser Sichtweise entsprechend geben allein die Naturwissenschaften Auskunft darüber, was Natur objektiv ausmacht, welche Strukturen und Prozesse in der Natur auftreten. Darüber hinaus gibt es subjektive Einstellungen und Zugänge zur Natur. Im Unterschied zu Naturwissenschaft haben diese Zugänge aber nicht Natur per se im Blick, sondern sind Ausdruck unseres Verhältnisses zur Natur.

Wenn auf der einen Seite die objektive (Erkenntnis von) Natur in den verschiedenen Wissenschaften wie Physik, Chemie und Biologie herrscht, finden sich auf der anderen Seite die subjektiven Einstellungen zur Natur, die zwischen den verschiedenen Zeitepochen, Kulturen und Individuen variieren. In dieser Sichtweise gibt es prinzipiell nur eine Natur - oft mit der »Welt« oder dem »Universum« identifiziert - und eine Form objektiver Naturerkenntnis. Aber es gibt verschiedene Zeitepochen, Kulturen und Individuen mit ihren unterschiedlichen Einstellungen zur Natur. Die variablen Formen »unseres« Verhältnisses zur Natur können natürlich ihrerseits Gegenstand der wissenschaftlichen Erforschung werden - etwa in den Geschichtswissenschaften, den Kunstwissenschaften oder der

\footnotetext{
* An dieser Stelle möchte ich meinen Dank für die finanzielle Unterstützung eines mit diesem Aufsatz inhaltlich verwandten Forschungsprojektes - Herman Dooyeweerd und Eric Voegelin: (Un-)zeitgemässe Denker der Transzendenz - durch den Schweizerischen Nationalfonds SNF äussern.

1 Dan Zahavi, Naturalized Phenomenology, in: Handbook of Phenomenology and Cognitive Science, hg. v. Shaun Gallagher/Daniel Schmicking, Mainz 2009, 2-19.
} 
verstehenden Soziologie. Doch sind diese Einstellungen zur Natur nicht Teil der Natur in derselben Weise, wie Kohlenstoffmoleküle, Spiralnebel oder Gehirnentzündungen "natürliche Phänomene« sind. Vielmehr verleihen sie der Natur einen - wie man sagt - erfahrbaren Sinn und Wert. Natur umfasst den Bereich empirisch-quantitativ messbarer, aber grundsätzlich wert- und sinnfreier Tatsachen; Kultur umfasst die Dimensionen von Sinn und Wert, die "wir« diesen Tatsachen beimessen oder nicht beimessen.

Soweit ein verbreitetes Verständnis der Dinge. Im Folgenden möchte ich dafür argumentieren, dass wir uns mit einem solchen Verständnis nicht zufriedengeben können oder sollten. Die Schwierigkeiten beginnen mit der Frage, wo Natur beginnt und wo sie aufhört. "Why make do with an unduly restricted conception of nature,${ }^{2}$ fragt Dan Zahavi. Warum Natur auf das reduzieren, was uns die gegenwärtigen Naturwissenschaften verraten können? Die Grenzen der Natur(-wissenschaften) sind nicht festgelegt. Mehr noch: diese Grenzen sind mit keinerlei empirischen Methoden oder mathematischen Formeln zu bestimmen. Das wird schnell ersichtlich, wenn wir uns folgende Fragen stellen: Wo endet Natur(wissenschaft)? Wo beginnt Kultur(-wissenschaft)? Wie ist das "Ganze« der Wirklichkeit beschaffen, von der Natur und Kultur Aspekte sind - einmal angenommen, diese Wirklichkeit umfasst weder nur Natur noch nur Kultur? Wenn wir solchen Fragen nachgehen, geraten wir unweigerlich auf philosophisches und theologisches Terrain. Was also soll unter "Natur" verstanden werden? Die griechischen Philosophen nannten die Natur to pan - das All. Je nach Zusammenhang ist ebenso von physis oder von kosmos die Rede. In biblischer Sprache heisst die Natur "Schöpfung». Weder das griechische All noch die biblische Schöpfung ist mit der modernen Auffassung von Natur identisch. Das folgt schon daraus, dass weder das eine noch das andere eine scharfe Abgrenzung zu Kultur und zum Göttlichen impliziert.

Der Philosoph, Theologe und Pädagoge Georg Picht (1913-1982) hat in seiner Vorlesung an der Universität Heidelberg zum Begriff der Natur und seiner Geschichte (1973-74) hervorgehoben, dass die moderne Auffassung von Natur durch einen Verzicht charakterisiert ist: "Die Naturwissenschaftler können ihre Forschungen nur deshalb betreiben, weil sie seit Galilei beschlossen haben, die unermesslich schwierige Frage, was sie zu ihren Erkenntnissen befähigt, auszuklammern. Sie fragen nicht nach der Natur überhaupt, weil sie entdeckt haben, dass der Verzicht (darauf), diese Frage zu stellen,

2 Ebd., 15. 
ihnen den Spielraum gibt, sich unbefangen der Erforschung von Phänomenen innerhalb der Natur zu widmen. $\ll^{3}$

Die Ironie an der Situation ist nicht zu verkennen. Die Naturwissenschaften haben das unbestrittene Monopol auf unser Verständnis von Natur. Gleichzeitig geben sie uns keine Antwort darauf - wie das einst Theologie und Philosophie taten - was Natur "überhaupt» ist oder was Natur zu Natur macht. "[J]ene Natur, die als umfassende und weise Macht, gleich einer Göttin alles Leben nach unwandelbaren Gesetzen zu erschaffen und zu lenken schien, die sich Dichtern und Denkern zu staunenderVerehrung bot, hat sich [...] aufgelöst. $\mathbb{1}^{4}$ Mehr noch: Die Frage, wie Einheit und Identität »der« Natur zu denken sind, gilt als unwissenschaftlich und darf in einem sich als wissenschaftlich verstehenden Zeitalter oft nicht einmal gestellt werden. ${ }^{5}$ An dieser Stelle könnte man freilich einwenden, dass es auf eine Antwort auf diese "unermesslich schwierige Frage nicht ankommt. Denn wenn keine naturwissenschaftliche Antwort möglich ist, muss es sich um eine "metaphysische" Frage handeln. Und darüber mögen sich diejenigen streiten, denen nichts Besseres einfällt als über Dinge nachzudenken, die zu ignorieren gemäss Hans Blumenberg gerade zum Erfolg moderner wissenschaftlicherVernunft und damit zur Legitimität menschlicher Selbstbehauptung gegenüber jedem philosophischen und theologischen Absolutismus beigetragen hat. ${ }^{6}$ Georg Picht hält in seiner Vorlesung dagegen - ohne direkt auf Blumenberg Bezug zu nehmen -, dass die unvermeidbare Kehrseite dieses unbestrittenen Erfolges des naturwissenschaftlichen Denkens und Forschens die Zerstörung der Natur ist. Gerade weil wir mit unseren Methoden richtig liegen, haben wir die Macht gewonnen, Natur zu erforschen und zu zerstören. ${ }^{7}$ So können für Picht wissenschaftliche Erkenntnisse zwar richtig sein, ohne deshalb wahr zu sein. Die Bedrohung der natürlichen Lebensgrundlagen unseres Daseins folgt aus der Bedrohung des modernen Denkens durch sich selbst - ein Denken, das sich vom Wahrheitsdenken im Sinn der griechischen physis und der biblischen "Schöpfung" weitgehend

\footnotetext{
3 Georg Picht, Der Begriff der Natur und seine Geschichte, Stuttgart 1989, 4.

4 Vgl. Walter Burkert, Kulte des Altertums. Biologische Grundlagen der Religion, München 1998, 9.

5 Streng genommen verbietet sich damit auch das naturalistische Programm einer "unity of science", demgemäss das "wahre "Wesen von Wissenschaft etwa in der Physik festgemacht wird.

6 Vgl. Hans Blumenberg, Die Legitimität der Neuzeit [1966], Frankfurt a. M. ${ }^{8} 2017$.

7 Nicht die physis der Griechen noch die biblische "Schöpfung können wir zerstören, aber die Biosphäre, die irdisches oder zumindest menschliches Leben trägt.
} 
emanzipiert hat. ${ }^{8}$ Daher steht es für den Heidelberger Philosophen nicht in unserem Belieben, die Frage nach der Einheit der Natur und ihrer Geschichte im westlichen Denken aufzuwerfen oder nicht aufzuwerfen. Die Frage ist nicht Ausdruck der Sehnsucht nach einem geschlossenen philosophischen System, sondern zielt darauf $a b$, welche Zukunft wir wollen und wie wir Verantwortung für sie übernehmen können.

Nun ist es nicht mein Ziel, den "Götterkampf« zwischen metaphysischen und anti-metaphysischen Denk- und Lebenseinstellungen, der heute quer durch die verschiedenen Wissenschaften, die Philosophie und die Theologie hindurchgeht, schlichten zu wollen. Es geht vielmehr darum zu verstehen, wie es zu diesem scheinbar unversöhnlichen Gegensatz zwischen anti-metaphysischem Frageverzicht und der metaphysischen Suche nach Letztbegründungen im Denken von Natur gekommen ist. Wenn wir unter Metaphysik jene Wissenschaft verstehen, "die zu erklären versuchte, wie es möglich ist, dass wir mit Hilfe der Wissenschaft Wahrheit erkennen «, tritt uns die Hintergründigkeit der skizzierten Situation vor Augen. Denn einerseits kann der Erfolg der modernen Naturwissenschaften nicht anders erklärt werden als dadurch, dass alles und jedes ihren experimentellen Methoden unterworfen wird..$^{10}$ Nichts von dem, was uns im Leben begegnet, nichts von dem, was wir erfahren, kann sich dem begrifflich objektivierenden Zugriff der Naturwissenschaften entziehen. Dadurch, so hatte schon ein Immanuel Kant gesehen, wird das traditionelle metaphysische Denken von Natur als physis oder als Schöpfung einer radikalen Infragestellung unterzogen - und damit unweigerlich auch die Stellung des Menschen im Universum und das Verhältnis beider zu Gott. ${ }^{11}$ Metaphysik und

\footnotetext{
8 Ohne diesen Punkt hier weiter ausführen zu können lässt sich doch sagen, dass in beiden Fällen Wahrheit als intaktes Verhältnis des Menschen zu Natur, zu Gott und zu sich selbst in den Blick kommt.

9 Picht, Der Begriff der Natur, 23.

10 "Wenn das Experiment zum Beweis einer Aussage genügt, ist die Wissenschaft der Mühe enthoben zu untersuchen, was sie unter Wahrheit versteht, wenn sie behauptet, diese Aussage sei wahr.« Ebd., 16.

${ }^{11}$ Dass damit kein simples "Konfliktmodell« zwischen Wissenschaft und Theologie impliziert ist, wie es eine überlieferte Weisheit seit dem späteren 19. Jahrhundert haben möchte, wird dann sichtbar, wenn die konstitutive Rolle von Religion und Theologie in der Legitimation der modernen Naturwissenschaften berücksichtigt wird.Vgl. Stephen Gaukroger, The Emergence of a Scientific Culture. Science and the Shaping of Modernity 1210-1685, Oxford 2006.
} 
Theologie sind fortan dem systematischen Verdacht ausgesetzt, es mit einer Art "Hinterwelt« zu tun zu haben. ${ }^{12}$

Andererseits aber, so zeigt sich im historischen Rückblick, stehen die modernen Naturwissenschaften trotz Wahrheitsverzicht ${ }^{13}$ dem Anspruch auf "absolutes" Wissen und Autorität der diskreditierten metaphysischen Tradition in Philosophie und Theologie in nichts nach. ${ }^{14}$ Gesellschaftliche Erwartungen, dass auf der Basis von Wissenschaft die Probleme der Menschheit gelöst werden können, übertreffen die Versprechen, die Philosophie und Religion für unser irdisches Leben gemacht haben, bei weitem. ${ }^{15}$ Und den globalisierten Folgen der modernen Naturwissenschaften in Technik und Industrie kann sich im Gegensatz zum Einfluss von Philosophie und Theologie niemand entziehen. Auf dem Gebiet der ihres traditionellen metaphysischen Fundamentes beraubten Philosophie selbst ist der Druck eines allumfassenden Naturalismus oder "Szientismus" eine kaum zu verkennende Tatsache. Allein, die Frage nach der Möglichkeit von Wahrheit menschlicher Erkenntnis und Daseinsgestaltung ist aus dem Bereich des naturwissenschaftlichen Denkens und Forschens herausgefallen und hat dort keine eigentliche Berechtigung mehr. Der Glaube an die Wahrheit von Erkenntnis - auch wissenschaftlicher Erkenntnis - ist wörtlich grundlos geworden. Nicht Skepsis oder theoretische Resignation, sondern gerade die Steigerung wissenschaftlicher Erkenntnisgewissheit und Machbarkeitserwartungen befördert den Nihilismus, den Nietzsche prophezeit hatte. ${ }^{16}$ Wir wissen, wie die Dinge funktionieren - was darauf hinweist, dass unsere Sicht auf die Dinge richtig ist -, aber wir wissen nicht, wie oder warum wir überhaupt etwas Wahres wissen oder erfahren können.

Wenn wir uns fragen, wie das moderne europäische Denken in diese eigentümliche Situation hineingeraten ist, kommen wir an Immanuel Kant nicht vorbei. Am einfachsten lässt sich das Problem, das Kant in aller Schärfe sichtbar gemacht hatte, vielleicht anhand der Rolle des "Subjekts" von Erfahrung und ihres "Gegenstandes" erläutern. Die Bedingungen der Möglichkeit von Erfahrung sind

\footnotetext{
${ }^{12}$ Vgl. Friedrich Nietzsche, z.B. Nachlass 1880-1882, in: KSA 9 (1999), 137. Die Suche nach der Möglichkeit einer nicht-metaphysischen Theologie sieht sich fortan dem Druck ausgesetzt, ein "weltloses« oder akosmisches Gottesverständnis zu entwickeln - oder sich umgekehrt als religiös verbrämte Sozialwissenschaft zu verstehen.

13 Oder, präziser, trotz dem Verzicht, sich auf die Voraussetzungen und die Möglichkeit ihres eigenen Wahrheitsanspruches einzulassen.

14 Picht, Der Begriff der Natur, 130.

15 Vgl. Gaukroger, The Emergence, 2.

${ }^{16}$ Picht, Der Begriff der Natur, 33.
} 
nach Kant bekanntlich im Erfahrungssubjekt und nicht in einer diesem Subjekt vorgegebenen Erfahrungswirklichkeit zu suchen. Die Gegenstände der "äusseren" Erfahrung werden durch zwei »innere" Quellen konstituiert: Sinnlichkeit und Verstand (plus Einbildungskraft). Die Kategorien des Verstandes bringen Einheit und Ordnung in die Mannigfaltigkeit der sinnlichen Eindrücke, die auf uns einwirken. Nur dadurch haben wir es überhaupt mit erfahrbaren Gegenständen zu tun, über die wir ein methodisch kontrolliertes Wissen gewinnen können. Die Einheit von Natur ist somit in der Einheit der logischen Verstandesfunktionen und Verstandesregeln begründet, deren Geltung den raumzeitlichen Gegenstand von Erfahrung allererst (mit)ermöglicht. So gesehen steht das Subjekt der Erfahrung in gewissem Sinn ausserhalb der Natur und dieser gegenüber. ${ }^{17}$ Natur wird zum Inbegriff der Gegenstände von möglicher äusserer Erfahrung. Der Geltungsgrund der Erfahrung aber liegt im »logischen Ich" des Erfahrungssubjekts verborgen. Was Natur "von sich aus" ist - oder wie sie "von sich her" erscheint -, entzieht sich unserem Erkenntnishorizont. Dennoch können wir vielleicht nicht anders, als diesen Horizont übersteigen zu wollen. Die Frage nach dem ureigenen Wesen von Natur und ihrem Urheber wird so zur unabweisbaren Quelle von »transzendentalem Schein«. Es ist dieser Sachverhalt, der Georg Picht in seiner Vorlesung zur These führt, dass der moderne Begriff von Natur die Natur zerstört - noch bevor Technik und Industrie zu messbaren Umweltbelastungen führen und wir das rücksichtslose Handeln allein an Macht oder Profit interessierter Akteure dafür verantwortlich machen könnten. Denn messbar werden diese Folgen ja allererst dadurch, dass sich die moderne naturwissenschaftliche Herangehensweise - deren wissenschaftlich unreflektierten Voraussetzungen den Keim der Zerstörung von Natur in sich enthalten - als richtig erwiesen hat.

Der Erfolg der modernen Naturwissenschaften beruht für Picht also darauf, dass das Subjekt der Erkenntnis aus der Natur "herausgebrochen ${ }^{18}{ }^{18}$ ist. Auf der einen Seite befinden sich die Objekte der äusseren Erfahrungswirklichkeit, die nach strengen Gesetzen von Ursache und Wirkung determiniert sind. Diesen Objekten gegenüber steht das nach inneren Gesetzen der Freiheit handelnde Subjekt, das sich dieser Objekte wissenschaftlich zu bemächtigen sucht. Dadurch aber gerät das Denken von Natur in eine grundlegende Antinomie. Denn das konkrete menschliche Subjekt ist

\footnotetext{
17 "In gewissem Sinn", weil die Cartesische Unterscheidung von res cogitans und res extensa im Horizont derVernunftkritik Kants keinen Substanzendualismus impliziert.

${ }^{18}$ Picht, Der Begriff der Natur, 389.
} 
eben nicht nur Erkenntnissubjekt, sondern zugleich auch Objekt seiner eigenen theoretischen Erkenntnisbemühungen. Wenn die Unterscheidung von Subjekt und Objekt auf dasjenige Wesen in der Natur angewendet wird, welches diese Unterscheidung hervorgebracht hat, gerät sie in abgründige Schwierigkeiten. Zugleich steht die Unterscheidung an der Basis des modernen wissenschaftlichen Denkens und Forschens und kann allein deshalb nicht aufgegeben werden. Das hat zur Folge, dass nicht nur die Einheit von Natur, sondern auch die Einheit der menschlichen Person verloren geht. Dem empirischen Subjekt - als Objekt der wissenschaftlichen Erforschung - steht fortan das transzendental-logische Subjekt Kants als Bedingung der Möglichkeit von Erkenntnis überhaupt gegenüber. Wie hängen die beiden aber zusammen? Diese Frage beschäftigt die Philosophie von Kant über Husserl bis in unsere Gegenwart und ist zur scheinbar unerschöpflichen Quelle von Perplexität und Missverständnis geworden.

In der Tat taucht die Frage auch heute - trotz aller Rede eines post-metaphysischen Zeitalters - immer wieder als Problem in der Gesellschaft auf. Die globalen Folgen dieser einst spezifisch europäischen Konstellation des Denkens sind kaum zu überschätzen. Wenn wir nach dem eigentlichen »Humanum» des Menschen fragen, oszillieren die Antworten fast unweigerlich zwischen einem "äusseren" und einem »inneren" Bereich. Inwiefern ist der Mensch Teil der natürlichen Welt? Lässt sich Bewusstsein als Bedingung von Freiheit naturalisieren? Welcher Begriff von Freiheit ist in allen wissenschaftlichen Versuchen einer Naturalisierung von Bewusstsein - egal ob erfolgreich oder nicht - notwendig vorausgesetzt? Die Grundkonstellation des Problems hält sich unter unzähligen Variationen durch: Das Verhalten »äusserer" Gegenstände - darunter etwa auch das menschliche Gehirn - ist durch kausale Notwendigkeit bestimmt; zugleich gibt es ohne die im Bewusstsein der Erkenntnissubjekte frei entworfenen Theorien und Experimente überhaupt kein wissenschaftlich verlässliches Wissen dieser Gegenstände und der sie bestimmenden Naturgesetze. ${ }^{19}$ Wenn aber der Mensch ein "natürliches« Wesen sein soll - und wer würde das bestreiten wollen? -, müssen die sich scheinbar ausschliessenden Pole von Freiheit und Notwendigkeit im Begriff der Natur zusammengedacht werden. Mit den herkömmlichen Mitteln der modernen Naturwissenschaften ist

\footnotetext{
${ }^{19}$ Dies gilt natürlich ebenso für reduktionistische Theorien des Bewusstseins, die jegliche Willensfreiheit mit wissenschaftlichen Argumenten ausschliessen wollen, wie für nichtreduktionistische Theorien.
} 
das nicht zu leisten. Das "wissenschaftliche Weltbild« steht hier vor einer recht oder schlecht verdeckten Aporie. ${ }^{20}$

Hinter dieser Aporie liegt für Georg Picht der Verlust der griechischen Natur als physis. Die Welt der Götter, der Menschen, Tiere und Pflanzen etc. bildete im griechischen Verständnis einen einzigen, umfassenden und unwandelbaren Tatsachen- und Sinnzusammenhang (»Identität«). Unsere Situation hat sich insofern verändert, als "für die Griechen die Identität die gesamte physis, einschliesslich des Menschen, zusammenhält, während in der Neuzeit der aus der Natur herausgebrochene Mensch sich selbst so stellen muss, als ob er die Identität aus sich selbst heraus ständig produzieren könnte. $\ll^{21}$ Trotz wiederkehrenden Versuchen in der europäischen Geschichte können wir aber nicht einfach zu einem solchen Verständnis von Natur zurückkehren. ${ }^{22}$

Nun ist es nicht so, dass Kant die mit seiner »kopernikanischen Wende" verbundenen Probleme nicht gesehen hätte. Sein eigener Versuch, die Einheit der menschlichen Person denkerisch zu gewährleisten, bestand darin, diese in der Einheit der Vernunft zu verankern. In diesem Zusammenhang spielen Kants so genannte "transzendentale Ideen" der Vernunft eine zentrale Rolle. Sie sind es, die die Bedingungen der Möglichkeit des richtigen Verstandesgebrauchs ausmachen. Ebenso wie der richtige - aber zunächst spontane - Gebrauch der logischen Funktionen des Verstandes die Erfahrung äusserer Gegenstände ermöglicht, ermöglichen die transzendentalen Ideen der Vernunft das, was bei Kant Verstand heisst. Durch die Vernunftideen werden also nicht die raumzeitlichen Gegenstände empirischer Erkenntnis, sondern die Möglichkeit wahrer Erkenntnis überhaupt konstituiert. ${ }^{23}$ Auf seine Weise nimmt Kant damit ein durch die gesamte metaphysische Tradition verlaufendes Motiv auf, wenn allein die Einheit der transzendentalen Ideen von Gott, Welt und Mensch (Seele) das "transzendental-logische Ich" oder "transzendentale Subjekt " konstituieren soll, das wiederum die Möglichkeit aller Erfahrung und der empirischen Erkenntnis verbürgt.

An dieser Stelle möchte ich auf einen weiteren Denker eingehen, der ebenso wie Georg Picht den grundlegenden Antinomien des

\footnotetext{
${ }^{20}$ Unmittelbar ersichtlich im Fall eines "materialistischen" Naturalismus, der die Bedingung der Möglichkeit seines eigenen Erfolges - das nach inneren Gesetzen der Freiheit handelnde Subjekt der wissenschaftlichen Erkenntnis - mit physikalischen Begriffen irgendwie zum Verschwinden bringen muss.

${ }^{21}$ Picht, Der Begriff der Natur, 389.

${ }^{22}$ Ebd., 196.

${ }^{23}$ Ebd., 218.
} 
modernen westlichen Denkens nachgegangen ist. Hermann Dooyeweerd (1896-1977) gehörte wie Picht zu den eher spärlich gesäten Philosophen des 20. Jh., die sich einerseits weit auf das theologische Denken der christlichen Traditionen eingelassen haben, andererseits aber dezidiert an der Autonomie der Philosophie gegenüber der Theologie festgehalten haben. ${ }^{24}$ Das ist umso erstaunlicher - und mag zumindest auf den ersten Blick paradox wirken -, als beide Denker die religiöse Dimension der Philosophie deutlich vor Augen hatten. Philosophie sieht sich seit ihren Anfängen mit der Frage nach dem Ganzen und dem Absoluten konfrontiert. "Absolute "Wahrheit ist kein theoretisch konstruierter oder spekulativ-abstrakter Begriff, sondern führt auf eine lebendige Erfahrung von "Göttlichkeit" zurück. An der Wurzel der griechischen Tradition philosophischen Denkens steht einerseits der Name des Parmenides für eine folgenreiche Epiphanie, die als Erschliessung des absoluten Seins-charakters von Erfahrung im "theoretischen" Denken umschrieben werden kann. Aus dieser Erfahrung heraus hat das Identitäts- bzw. SubstanzDenken konstitutiven Charakter für die europäische Tradition angenommen:Alles, was wahrhaft ist, ist mit sich selbst identisch, existiert unabhängig vom Bezug zum "Anderen«. Die göttliche Sphäre des reinen Seins bzw. des reinen Denkens schliesst jeden Wandel aus.

Nicht weniger bedeutsam für das europäische Denken ist der Name Heraklits. Auch dieser geht von einer "religiösen « Erfahrung aus, die aufs Ganze der menschlichen Erfahrungswirklichkeit zielt. Aber diese Erfahrung ist gewissermassen die Antipode zur eben geschilderten. Sie hat die ewige Wechselbewegung und das ewige Gleichgewicht von Entstehen und Vergehen alles Seienden im Blick. Das Entscheidende liegt für Dooyeweerd nun darin, dass diese beiden Erfahrungen im Anfang des philosophischen Denkens keine psychischen Zufälligkeiten oder historisch bedingten Eigenschaften ihrer jeweiligen Träger beschreiben, sondern epochalen Charakter besitzen. Ohne auf die zahlreichen philologischen Vertracktheiten und hermeneutischen Komplexitäten der einschlägigen Debatten einzugehen, soll hier bloss festgehalten werden, dass Dooyeweerd in der Wurzel des griechisch-philosophischen Denkens eine religiöse Dialektik am Werk sieht. Der religiöse Charakter dieser Dialektik ist dafür verantwortlich, dass jeder Versuch einer theoretisch-wissenschaftlichen Synthese scheitert. ${ }^{25}$ Die beiden genannten Erfahrungen

\footnotetext{
${ }^{24}$ Als protestantische Denker weisen beide die mittelalterliche Auffassung von Theologie als der "regina scientorum« (Königin der Wissenschaften) zurück.

${ }^{25}$ Vgl. Herman Dooyeweerd, Reformation and Scholasticism in Philosophy. Volume 1:The Greek Prelude (Series A,Volume 5), Grand Rapids 2012, 9. Ganz ähnlich Picht:
} 
von (ewiger) Selbst-Identität und (ewigem) Wandel haben vielmehr die unweigerliche Tendenz, sich im menschlichen Bewusstsein zu verabsolutieren und die gegenläufige Erfahrung auszublenden oder in ihrer Geltung und Reichweite zu beschneiden. Dies hat Folgen für das philosophische und wissenschaftliche Denken von der Antike bis ins 20. Jh.

An der Basis eines jeden philosophischen und wissenschaftlichen Wirklichkeitsverständnisses steht für Dooyeweerd ein religiöses »Grundmotiv«. Damit gemeint ist eine spirituelle Dynamik, die das menschliche Leben insgesamt und das theoretisch-begriffliche Denken im Besonderen in bestimmte Bahnen lenkt. So wie wissenschaftliche Theorien durch empirische Messdaten unterdeterminiert sind, sind umfassende Welt- und Menschenbilder durch wissenschaftliche und philosophische Theorien unterdeterminiert. Trotz oder vielleicht wegen ihrer vortheoretischen Anziehungskraft prägen religiöse Grundmotive den "Geist» einer geschichtlichen Epoche. Das griechische Grundmotiv von Materie und Form umfasst zwei einander dialektisch entgegengesetzte Prinzipien. Diese legen die ursprünglichen Rahmenbedingungen des europäischen Denkens und die "Logik» der theoretischen Begriffsbildung fest. ${ }^{26}$ Auf der einen Seite steht das Prinzip der Materie. Dieses entstammt der reflexiven Aneignung der mythischen Vorstellung eines ewigen Lebensstroms, aus dem alles Geformte und Begrenzte vorübergehend heraustritt, um schliesslich wieder darin aufzugehen. ${ }^{27}$ Symbolisiert wird dieses "irrationale " Prinzip durch das Rad von Geburt, Wachstum, Reife, Verfall, Tod und Wiedergeburt. In diesem so unerbittlichen wie unberechenbaren Kreislauf leisten die Dinge einander "Gerechtigkeit« (dikē), indem das eine dem anderen Platz

»Es besteht schon zwischen Heraklit und Parmenides eine Inkommensurabilität, die selbst Platons ungeheuren Versuch einer Synthese dieser beiden Entwürfe nicht aufzulösen vermochte [...] Die Inkommensurabilität der Entwürfe, die der heutigen Wissenschaft zugrunde liegen, übersetzt sich aber in dieser Wissenschaft selbst in Antinomien, die durch methodische Äquilibristik nicht ausgeglichen werden können.» (319)

${ }^{26}$ Die sprachlichen Ausdrücke »Form« (morphē) und »Materie« (hylē) sind durch Aristoteles zum festen Bestandteil des philosophischen Vokabulars geworden. Die damit bezeugte Sache geht aber viel weiter zurück, obwohl die Vorstufen dieses Grundmotivs ausserhalb Hellas im historischen Halbdunkel liegen und bis heute Gegenstand von religionshistorischer Spekulation bleiben.Vgl. Walter Burkert, Griechische Religion der archaischen und klassischen Epoche [1977], Stuttgart ${ }^{2} 2011$, für einen aktuellen Überblick.

${ }^{27}$ Es sollte deutlich sein, dass diese Auffassung von "Materie« nicht viel gemeinsam hat mit Materie als Inbegriff von festen, "physischen« Bestandteilen der Erfahrungswirklichkeit. 
macht. Nichts währt ewig. Wie Ebbe und Flut sind die Dinge des menschlichen Lebens und alle natürlichen Phänomene von einem steten Auf und Ab gekennzeichnet. Im Geltungsbereich der »Materie" herrscht die Anankē oder "schicksalshafte Notwendigkeit", die auf der Ebene des einzelnen Lebewesens ebenso »blinder Zufall« heissen kann. Auf der anderen Seite der Erfahrung steht das "rationale" Prinzip der Form. Dieses zeichnet sich verantwortlich für den geordneten Bestand alles Seienden in der Zeit. Ausdruck davon ist die Rolle des nous (Vernunft) oder logos, der allem, was ist, nicht nur Form und Identität, sondern eben auch rationale Ordnung und Intelligibilität verleiht.

Das im Verlauf der Zeit immer komplexere Ineinandergreifen der beiden Prinzipien von Materie und Form ist in Platos Darstellung der Entstehung von Zeit (chronos) in der späten Schrift Timaios anschaulich geschildert. ${ }^{28}$ Als der "Vater" (=nous) das All erzeugte, verlieh er diesem eine feste Ordnung, indem er ein "nach der Vielheit der Zahl sich fortbewegendes dauerndes Abbild" seiner "in der Einheit beharrenden Ewigkeit « - eben die Zeit - schuf. Zusammen mit dem All als einem immerwährenden, beseelten und stofflichen Wesen ist auch die Zeit entstanden. Es fällt nun auf, dass Plato - im Gegensatz zu Newton - die Bewegung der Zeit als kreisförmige darstellt. Aber diese Bewegung ist hier der Kreisbewegung der rationalen Weltseele nachempfunden und nicht etwa dem Kreislauf von Werden und Vergehen des Materiemotivs. Denn Weltseele und Zeit haben ihren Ursprung in der ewigen Formkraft des göttlichen nous und nicht in der amorphen physis.

Die beiden Prinzipien von Materie und Form sind für Dooyeweerd zuerst und zunächst keine theoretischen "Positionen " oder Setzungen - auch wenn sie sich unweigerlich im theoretischen Denken ausdrücken und dort in begrifflich verfestigter Form greifbar werden. ${ }^{29}$ Sie lassen sich vielmehr zurückverfolgen bis in die mythische Welt der Götter und Heroen. Im Kontext des vorphilosophischen Mythos der antiken griechischen Welt sind den beiden Prinzipien die entsprechenden Gottheiten zugeordnet, allen voran Dionysos (Naturgottheit) und Apollo (Kulturgottheit). ${ }^{30}$ Obwohl

\footnotetext{
${ }^{28}$ Vgl. Dooyeweerd, Reformation and Scholasticism I, 297.

${ }^{29}$ Locus classicus dafür ist die "Metaphysik« des Aristoteles.

30 "Mit solchem Verfliessen der persönlichen Geformtheit steht der Dionysos-Kult in Kontrast zu dem, was mit Recht als typisch griechisch gilt; wieso eben darum beides, das `Apollinische und das `Dionysische〈, als Polarität zusammengehören, ist eine Frage der Kulturpsychologie, die genial und eigenwillig Friedrich Nietzsche gestellt hat."Burkert, Griechische Religion, 249f. Dooyeweerd bringt seine Einsicht in den ungelösten religiösen Konflikt an der Wurzel des griechischen Denkens denn
} 
diese verschiedenen Göttergestalten durchaus Züge des jeweils anderen annehmen können, wird deren wesensmässige Unvereinbarkeit dadurch symbolisiert, dass die olympischen Götter »Mutter Erde « verlassen. ${ }^{31}$ Entsprechend hat auch Zeus, der höchste Gott auf dem Berg Olymp, keine Macht über das Reich der Anankēe. ${ }^{32}$ Der sterbliche Teil der menschlichen Seele muss wie jedes vergängliche Lebewesen zurück in den Hades (Unterwelt).

Charakteristisch für das griechische und jedes »dialektische« religiöse Grundmotiv sind gemäss Dooyeweerd also zwei Dinge. Erstens bedingen die beiden in ihm vereinten Prinzipien einander. Keines tritt in der menschlichen Erfahrungswirklichkeit ohne das jeweils andere auf. Zweitens sind die beiden Prinzipien inkommensurabel. Zwischen ihnen lässt sich ersichtlich kein reflexives Gleichgewicht herstellen. Eines hat immer die Oberhand. Im Verlauf der Zeit verdrängt der philosophische Logos (Formmotiv) so zunehmend den vorphilosophischen Mythos (Materiemotiv), ohne diesen wirklich überwinden zu können. Die menschliche Erfahrungswirklichkeit widersetzt sich einer durchgängigen "Rationalisierung durch die göttliche bzw. vergöttlichte Vernunft. Zurück bleibt die gesellschaftliche Entfremdung der "irrationalen" Massen von den führenden Eliten sowie ein residualer Dualismus der grundlegenden Prinzipien des theoretischen Denkens, auch wenn dieser Dualismus durch begriffliche Dialektik oft getarnt ist.

Diese knappen Ausführungen sind nötig, um Dooyeweerds systematisch-philosophischen Denkansatz zu verstehen. ${ }^{33}$ Wie erwähnt sieht sich der niederländische Rechtsgelehrte und Philosoph vor die Aufgabe gestellt, das wissenschaftliche und philosophische Denken seiner Zeit mit der eigenen Verwurzelung in der christlichen Glaubenstradition reformiert-calvinistischer Ausprägung in ein kohärentes Verhältnis zu bringen. Wie konnte das gelingen? Ohne die ihm eigene Originalität zu verkennen, kann die "Kehre« ${ }^{34}$ d.h. die Abwendung vom Synthese-Gedanken in Dooyeweerds phi-

auch explizit mit dem "genialen Jugendwerk« Nietzsches, Die Geburt der Tragödie aus dem Geist der Musik, in Verbindung. Vgl. Dooyeweerd, Reformation and Scholasticism, 4.

${ }^{31}$ Ebd., 8.

${ }^{32}$ Oder setzt seine Macht mit Rücksicht auf die anderen Götter an der Grenze des Todes zumindest nicht ein, vgl. Burkert, Griechische Religion, 204.

${ }^{33}$ Das dreibändige Hauptwerk Dooyeweerds trägt den Titel De Wijsbegeerte der Wetsidee, Amsterdam 1935-36, ins Amerikanische übersetzt und erweitert in New Critique of Theoretical Thought, 4 Bde., Lewiston 1997.

${ }^{34} \mathrm{Zu}$ diesem "great turning point « vgl. Dooyeweerd, New Critique of Theoretical Thought I, v. 
losophischer Entwicklung als Wiederaufnahme des augustinischen Gedankens einer "philosophia christiana" unter radikal veränderten historischen Bedingungen - und mit viel Kant und Husserl im Gepäck - bezeichnet werden. Entgegen einer bis heute vorherrschenden "aufgeklärten" Auffassung konnte für ihn das "säkulare" Denken und Forschen an den modernen Universitäten nicht länger als weltanschaulich-religiös "neutral" durchgehen. Andererseits mochte er sich auch nicht in einen weltanschaulich-religiösen Fideismus retten, dem jegliche theoretische Auseinandersetzung mit den Errungenschaften der modernen Wissenschaften und den philosophischen Entwicklungen der Zeit als überflüssig oder gar heilsbedrohlich gelten musste. Denn mit der epochalen Verbreitung des christlichen Glaubens in der hellenistischen Welt war da noch ein anderes, namentlich biblisches, religiöses Grundmotiv auf die geistige und politische Weltbühne getreten: das Grundmotiv von Schöpfung, Fall und Erlösung in Jesus Christus in Gemeinschaft mit dem heiligen Geist. Auch dieses in der Reformation des 16. Jh. (zumindest parziell) wiederentdeckte Grundmotiv umfasst für Dooyeweerd das Ganze der Erfahrung und setzt eine eigene Logik der Begriffsbildung aus sich heraus - und dies nicht nur in einem religiösen oder kirchlich-theologischen Bereich, sondern potenziell in allen Bereichen des Lebens und des Denkens. ${ }^{35}$ Die Konfrontation mit der vorherrschenden Idee einer "autonomen "Vernunft war vorprogrammiert. Weil das biblische mit dem griechischen Grundmotiv in einen äusserst vielschichtigen und tief kulturprägenden Interaktionsprozess geraten war - der imVerlauf der Jahrhunderte zwei weitere Grundmotive hervorgebracht hatte -, vermochte ihn die Leitvorstellung einer autonomen theoretischen Vernunft nicht länger zu überzeugen. Denn je nach zugrunde liegendem Grundmotiv musste diese "Autonomie« sehr unterschiedliche Dinge bedeuten.

Zunächst war aus der Begegnung von "Athen" und "Jerusalem" in der christlichen Spätantike das für die gesamte mittelalterliche Epoche formative, scholastische Grundmotiv von Natur und Gnade hervorgegangen. Hier kommt dem Pol der »Natur" eine ganz spezifische Form von Autonomie zu, insofern "Natur« als endliches Medium für den Aufstieg der Seele zum unendlichen Gott und den Empfang der göttlichen Gnade dient. Im Unterschied zur innerhalb des griechischen Formmotivs gedachten Natur und der philosophischen theoria kann und muss die menschliche Natur diesen Aufstieg zum göttlichen Ursprung der Erfahrungswirklichkeit nicht aus sich

${ }_{35}$ Deshalb der Gedanke einer christlichen Philosophie und nicht bloss einer christlichen Theologie, die gemäss Dooyeweerd zu den "positiven"Wissenschaften zählte. 
selbst bewirken. Gegenüber der rettenden Heilsgegenwart Gottes - vermittelt durch die kirchlichen Sakramente - besitzt "Natur" vielmehr eine kreatürliche Autonomie. Durch die grundstürzende "Umbesetzung« des mittelalterlichen Motivs von Natur und Gnade am Vorabend der Moderne war sodann das Grundmotiv von Natur und Freiheit entstanden, welches zum philosophischen Ausgangspunkt eines Immanuel Kant wurde. Beiden dieser nachfolgenden Grundmotive gemeinsam ist der Versuch einer epochalen Synthese. Das scholastische Motiv von Natur und Gnade findet den Höhepunkt seiner begrifflichen Ausarbeitung bei Thomas von Aquin, der das biblische mit dem griechischen Motiv in einer umfassenden Synthese zu versöhnen suchte. Das humanistische Motiv von Natur und Freiheit stellt wiederum den Versuch dar, alle vorausgehenden Grundmotive im Horizont des modernen Subjekt- und Fortschrittsdenkens zu verbinden. Das Resultat dieser - für Dooyeweerd notwendig gescheiterten - Versuche zu Beginn des 20. Jh. ist die "postmoderne" Vielfalt philosophischer Ausgangspunkte des Denkens und Forschens. Sie alle halten sich für "evident", da scheinbar in einer zeitlosen, allgemein-menschlichen Vernunft begründet. Ihnen gegenüber steht eine wachsende Zahl anti-philosophischer Ausgangspunkte, die die Verabsolutierung »der«Vernunft zu einem universalen, alles beherrschenden Prinzip unterlaufen wollen. Auf diesem Hintergrund war die Idee einer autonomen Vernunft für Dooyeweerd zu einem blossen Dogma geworden, das nach einer erneuten "transzendentalen Kritik " verlangte.

Damit schliesst sich der Kreis in diesem Aufsatz. Die philosophische Herausforderung, der sich Herman Dooyeweerd vielleicht noch dezidierter als Georg Picht gestellt hatte, und die heute kaum weniger aktuell ist als vor fünfzig Jahren, lautet: Wie können wir einen Begriff von Natur entwickeln, in dem keiner der verschiedenen Aspekte menschlicher Erfahrungswirklichkeit - physische, biotische, psychische, logische, historische, ökonomische, rechtliche und ethische Aspekte - zu Ungunsten eines anderen theoretisch verabsolutiert wird? Wie kann es gelingen, alle diese Aspekte - Natur und Kultur - als kompossibel und gleichermassen real zu denken? Ist dies auf dem Boden eines rein immanenten Verständnisses von "Natur" überhaupt möglich, oder braucht es dazu eine Idee von Transzendenz? Wie auch immer wir diese Fragen beantworten, eines dürfte doch klar geworden sein: Die gegenwärtigen Bestrebungen einer wissenschaftlichen "Naturalisierung" von Bewusstsein und Denken setzen eine durch und durch theoretisierte Natur voraus. Nicht anders als bei Kant - aber ohne dessen kritisches philosophisches Bewusst- 
sein - scheint die Einheit und Identität von Natur einmal mehr in derVerabsolutierung der logischen Funktionen des Verstandes zu liegen.

Ich möchte diesen Beitrag deshalb nicht beenden, ohne die positive Stossrichtung des Denkansatzes Herman Dooyeweerds für unser Thema knapp zu skizzieren. ${ }^{36}$ An erster Stelle ist anzumerken, dass die christliche Philosophie Dooyeweerds und der Reformational Philosophy insgesamt den Anspruch hat, jeden theoretischen Reduktionismus in Wissenschaft und Philosophie zu vermeiden. ${ }^{37}$ Damit verbunden ist eine Umstellung der Grundkonstellation neuzeitlichen Denkens. Der Mensch kommt philosophisch nicht - wie bei Descartes und seinen Nachfolgern - primär als theoretisch-epistemisches Subjekt in den Blick, das sich der Aussenwelt als einem Objekt gegenübersieht. Vielmehr ist der Mensch primär Subjekt "gewöhnlicher Erfahrung ${ }^{38}{ }^{38}$ Wissenschaftliche und philosophische Theoriebildungs- und Reflexionsprozesse sind somit Bestandteil einer sich innerhalb dieser Erfahrung ausdifferenzierenden epistemischen Praxis. Es ist die gewöhnliche Erfahrung, welche die theoretisch-begriffliche Erfahrung trägt und nicht umgekehrt. ${ }^{39}$ Die Idee einer "freistehenden", theoretisch-begrifflichen Vernunft ist unter dem Vorzeichen einer modernen, ausdifferenzierten Gesellschaftsordnung eine zwar unvermeidliche Abstraktion - aber eben doch eine Abstraktion. Getragen wird diese Abstraktion durch ein Kontinuum menschlicher Erfahrungsfunktionen, unter denen der logisch-analytische Aspekt der Erfahrung bloss einer neben anderen ist. Menschliche Erfahrung hat nicht bloss logischen, sondern immer auch physischen, biotischen, sinnlich-sensorischen, emotionalen, sprachlichen, historischen, sozialen, ästhetischen usw. Charakter. Theoretische Reduktionismen zeichnen sich nun dadurch aus, dass

\footnotetext{
${ }^{36}$ Eine zugängliche Einfuihrung in diesen Denkansatz bietet Roy Clouser, The Myth of Religious Neutrality, Notre Dame 2005.

${ }^{37}$ Diesen Anspruch teilt sie mit anderen philosophischen Richtungen wie der Phänomenologie und der Tradition des amerikanischen Pragmatismus. Damit ist freilich nicht die Ablehnung eines methodischen Reduktionismus - z.B. die Integration einer wissenschaftlichen Theorie in eine umfassendere Theorie innerhalb desselben Gegenstandsbereichs - gemeint, sondern der ontologische Anspruch einer jeden reduktiven Theorie und Weltanschauung zurückgewiesen.

${ }^{38}$ Ich entnehme diesen Ausdruck der gleichnamigen Publikation von Matthias Jung, Gewöhnliche Erfahrung, Tübingen 2014.

${ }^{39}$ Das bedeutet freilich nicht, dass vortheoretische Erfahrung gegenüber wissenschaftlicher und philosophischer Kritik immun ist, sondern nur, dass theoretische Erfahrung immer auf vortheoretische Erfahrung angewiesen bleibt.
} 
sie einen oder mehrere dieser Aspekte von allen anderen isolieren bzw. verabsolutieren möchten.

Die frühe Umstellung auf gewöhnliche Erfahrung macht die Reformational Philosophy gerade in der heutigen Situation, in der immer mehr Stimmen in Wissenschaft und Philosophie auf eine dezidierte Abkehr vom reduktionistischen Programm der unity-of-science des 20. Jh. hin zu einer pluralistischen Ontologie der Wissenschaften drängen, zu einem äusserst interessanten Gesprächspartner - nicht zuletzt für die Theologie..$^{40}$ Der biblische Gedanke von Schöpfung widersetzt sich für Dooyeweerd einem theoretischen Reduktionismus kategorisch. Der Grund dafür liegt wie bereits erwähnt in der Tatsache, dass derjenige Aspekt der vortheoretischen Erfahrungswirklichkeit (z.B. der physische Aspekt), auf den ein anderer Aspekt der Erfahrung (z.B. der biotische oder der sinnlich-sensorische) theoretisch zurückgeführt werden soll, verabsolutiert wird und dadurch gottähnlichen Status annimmt. In theistisch-religiöser Sprache kommt dies einem Akt der Idolatrie gleich. Der Schöpfer des Universums wird den Gesetzen eines bestimmten geschöpflichen Erfahrungsaspekts unterworfen.

Die philosophische Einforderung des Freiheits- und Souveränitätsanspruchs Gottes gegenüber seiner Schöpfung in all ihren Aspekten hat aber nicht bloss die religiöse Funktion der Wahrung des biblischen Idolatrieverbotes. Untrennbar damit verbunden ist die Wahrung der Fülle der lebensweltlichen Erfahrungswirklichkeit, innerhalb derer sich das menschliche wie alles andere kreatürliche Leben entwickeln und gedeihen soll. Kreatürliches Leben wird immer dort eingeschränkt, wo es theoretisch-begrifflich auf die (Vor-) Bedingungen seines Auftretens reduziert wird. Wir wissen, dass es kein menschliches Fühlen, Denken und Wollen gibt ohne neuronale Aktivität des Gehirns. Daraus folgt aber nicht, dass Bewusstsein nichts anderes ist als das Resultat biotischer Hirnfunktionen und diese wiederum nur ein Epiphänomen der physisch-chemischen Substratfunktionen eines bestimmten Körperteils sind. Der Physikalismus, der in einer entsprechenden Auffassung von Bewusstsein und Denken zum Tragen kommt, hat keinen wissenschaftlichen Charakter im engeren Sinn, sondern ist Bestandteil eines szientistisch-weltanschaulichen commitment einer bestimmten Forschergemeinschaft. Da keine Wissenschaft ohne umfassenden Deutehorizont in Form eines mehr oder weniger artikulierten »Weltbildes« auskommt, ist

\footnotetext{
${ }^{40}$ Dies umso mehr, als in dieser philosophischen Tradition auch die Einseitigkeiten einer "hermeneutischen Philosophie» und deren Dichotomie zwischen (Natur-)Erklärung und einem »reinen« (Sinn-)Verstehen vermieden werden.
} 
diese Option natürlich nicht a priori illegitim. Nur darf sie nicht als Teil oder notwendige Folge von Wissenschaft ausgegeben werden.

Der ontologische Status, der dem physischen Aspekt von Erfahrung in einem physikalistischen Denkhorizont zukommt, ist für Dooyeweerd analog zum Status, den Gott in einem theistischreligiösen Denkhorizont einnimmt. Dasselbe gilt für die Idee eines transzendental-logischen Subjekts. Metaphorisch könnte dieser Status als "Quelle« aller Erfahrung bezeichnet werden - etwas weniger metaphorisch als unhintergehbarer Ermöglichungsgrund aller zeitlichen Akte und Korrelate von gewöhnlicher Erfahrung. Deshalb sind Physikalismus und transzendentaler Logizismus für diesen Denker nicht religiös neutral, sondern Ausdruck eines immanent-religiösen Grundmotivs. In seinem vom biblischen Grundmotiv geleiteten Denkansatz hingegen sind alle Aspekte der vortheoretischen Erfahrung ontologisch gleichermassen robust. Daraus folgt, dass die den verschiedenen Erfahrungsaspekten ${ }^{41}$ zugeordneten Wissenschaften allesamt "harte (oder "weiche«) Wissenschaften sind - von der Physik bis zur Theologie! Deren Zusammenhang liegt darin, dass die ihnen entsprechenden Erfahrungsaspekte durch ein unumkehrbares ontisches Voraussetzungsverhältnis verbunden sind. Der biotische Aspekt setzt den physischen Aspekt voraus und ist seinerseits Voraussetzung für alle nachfolgenden Aspekte - bis hin zum fiduziären Aspekt ("Vertrauen«), der für die Theologie eine konstitutive Rolle spielt. Der Unterschied zwischen den verschiedenen Erfahrungsaspekten liegt wiederum darin, dass die sie jeweils auszeichnenden irreduziblen "Gesetze« oder ontischen Ermöglichungsbedingungen in dieser Ordnung immer mehr normativen und immer weniger determinierenden Charakter besitzen. Die Gesetze des biotischen Aspekts, wie sie primär in Prozessen des organischen Lebens zum Ausdruck kommen, sind in dieser Hinsicht besonders interessant. Einerseits sind solche Prozesse (z.B. Stoffwechsel) durch determinierende "Gesetze« bestimmt, die keine Abweichung erlauben bzw. deren Inhibition zum Tod des biotischen Subjekts führt. Andererseits unterstehen solche Prozesse aber auch normierenden Gesetzen, die bestimmte Freiheitsgrade der Entwicklung und des Verhaltens ermöglichen. Diese Freiheitsgrade weiten sich in den post-biotischen

\footnotetext{
${ }^{41}$ Dooyeweerd unterscheidet derer fünfzehn, wobei die Liste so fallibel wie korrigibel ist. Auf die Kriterien der Identifikation genuin irreduzibler Erfahrungsaspekte kann hier aus Platzgründen nicht eingegangen werden.Vgl. H. Dooyeweerd, Die Philosophie der Gesetzesidee und ihre Bedeutung für die Rechts- und Sozialphilosophie, in: Archiv für Rechts- und Sozialphilosophie 53 (1967), Nr. 3 \& 4, 1-30 \& 465-513, 7.
} 
Aspekten der Erfahrung entsprechend der Art der involvierten Gesetze und Normen beständig aus. Ökonomische Normen können, wenn auch nicht beliebig, deformiert oder missachtet werden ${ }^{42}$ moralische und fiduziäre Normen (»Vertrauen«) ebenso. In religiöser Hinsicht - wodurch das Ganze der Erfahrung in den Blick kommt - können sich Vertrauensnormen wie gesehen in sehr verschiedenen Grundmotiven ausdrücken.

Auffällig an diesem Denkansatz ist die Tatsache, dass die verschiedenen Aspekte der Erfahrung sowohl irreduzibel als auch voneinander untrennbar sind. Menschliche Erfahrungssubjekte bilden aktive Funktionen in allen Aspekten der Erfahrungswirklichkeit aus. An diesem Punkt zeigt sich die universale Geltung dieser Aspekte im Kontrast zu partikularen Erfahrungsdingen (Subjekte oder Objekte) am deutlichsten. Beispielsweise kommt niemand darum herum, sich zur Vertrauenswürdigkeit anderer Personen oder zur Verlässlichkeit von Wahrnehmungsobjekten insgesamt zu verhalten. Als Grenzfall von Vertrauen lässt sich das auch vom Ganzen der Erfahrung sagen. Aber niemand ist genötigt, diesem oder jenem Menschen zu vertrauen oder einem bestimmten religiösen Grundmotiv zu folgen. Man muss sich auch nicht für konkrete gesellschaftshistorische Entwicklungen interessieren, obwohl wir alle bewusst oder unbewusst von Gesellschaft und Geschichte geprägt sind. Daraus folgt, dass Erfahrungsaspekte nicht mit faktischen Erfahrungsdingen verwechselt werden dürfen. Letztere betreffen das Was, erstere das Wie von Erfahrung.

Nun sind nicht nur Menschen, sondern alles, was uns begegnet, jedes zeitliche Phänomen in Natur und Kultur, durch die verschiedenen Aspekte der Erfahrung geprägt. Es gibt keine "rein" physischen Dinge oder "rein " logische Entitäten auf dem Boden der gewöhnlichen Erfahrung. Wir können unsere theoretischbegriffliche Aufmerksamkeit zwar auf die physischen, sensorischen, logischen oder rechtlichen Bedingungen des Auftretens bestimmter Phänomene richten und von allen anderen Bedingungen abstrahieren. Dadurch erweitert sich unser Handlungsspielraum in wahrhaft dramatischer Weise. Aber liessen sich "rein« physische Dinge logisch nicht auseinanderhalten oder "rein« logische Entitäten wie Propositionen und Theorien sprachlich nicht identifizieren, würden sie sich der gewöhnlichen Erfahrung nicht erschliessen. Dann könnten wir

\footnotetext{
42 Ökonomisch bestimmte Unternehmen können Prinzipien der Profitabilität missachten und ihr Fortbestehen auf anderem Weg sichern, z.B. durch (legale) Querfinanzierung oder (illegale) Extorsion.
} 
auch keine wissenschaftlichen oder philosophischen Theorien über solche Dinge aufstellen.

Kurzum, in der gewöhnlichen Erfahrung sind alle verschiedenen Aspekte vereint. Zugespitzt gesagt: alles ist Natur und alles ist Kultur.Voraussetzung für diese holistische Sichtweise ist, dass aktive Subjektfunktionen von passiven Objektfunktionen unterschieden werden. Unsere physische Umgebung unterscheidet sich nicht von selbst in sensorisch, logisch, historisch oder rechtlich distinkte Objekte. Dazu braucht es sensorische etc. Subjekte, die bestimmte Objektfunktionen ihrer physischen Umgebung bestimmter Gesetze und Normen entsprechend realisieren. Die Unterscheidung zwischen aktiven Subjekt- und passiven Objektfunktionen lässt sich am Beispiel aktueller Theorien einer embodied cognition nochmals verdeutlichen. Folgen wir dieser wissenschaftlichen Stossrichtung, lässt sich das Phänomen des menschlichen Bewusstseins nicht als Resultat physisch-chemischer Hirnfunktionen reduzieren. Bewusstsein setzt neben einem beweglichen Leib auch eine physische Umgebung voraus, deren sinnlich-sensorische Objektfunktionen durch einen sensorisch begabten Organismus realisiert werden können. ${ }^{43}$

Alles ist Natur und alles ist Kultur. Aber Natur und Kultur sind nicht alles. Deren untrennbare Einheit und irreduzible Unterschiedenheit liegt gemäss dem hier skizzierten Denkansatz im menschlichen Erfahrungssubjekt als Geschöpf Gottes begründet, das sich weder auf seine natürlichen noch auf seine kulturellen Aspekte reduzieren lässt, sondern auf ein Ganzes der Erfahrung bezogen ist, dessen göttlicher Ermöglichungsgrund alle Aspekte der Erfahrung transzendiert.

- PD Dr. Johannes Corrodi Katzenstein ist Privatdozent für Religionsphilosophie am Institut für Hermeneutik und Religionsphilosophie (IHR) der Theologischen Fakultät der Universität Zürich.

${ }^{43}$ Vgl. dazu Alva R. Noë, Out of Our Heads. Why You Are Not Your Brain, and Other Lessons from the Biology of Consciousness, New York 2009. 